Accelerator Division

Alternating Gradient Synchrotron Department BROOKHAVEN NATIONAL LABORATORY

Upton, N.Y. 11973

Accelerator Division Technical Note

AGS/AD/Tech. Note No. 398

\title{
Recursive Transverse Damping Algorithms
}

\author{
T.Roser
}

August 11, 1994

\begin{abstract}
Recursive damping algorithms allow for a simple and fast closed orbit subtraction with a long and also adjustable integration time. A long integration time is necessary for operating a transverse damper with a betatron tune value near an integer. A simple implementation as a digital filter is discussed.
\end{abstract}




\section{Introduction}

The Booster and AGS transverse damper use one pick-up electrode (PUE) to measure the bunch position and a strip line to apply a correcting kick to the same bunch on the following turn. The magnitude of the kick has to be determined such that the coherent dipole motion of the bunch is reduced. In this technical note, I am extending the formalism described in Tech. Note 377[?] to include the situation when the PUE is not at the same position as the strip line kicker and, more importantly, also extend the digital filter design to include recursive filters. A recursive filter design allows for the implementation of a efficient and flexible closed orbit correction scheme.

\section{Damping Algorithm}

The amount of coherent dipole motion of the bunch is expressed in terms of the CourantSnyder invariant:

$$
\epsilon=\pi\left(\gamma x_{k}^{2}+2 \alpha x_{k} x_{k}^{\prime}+\beta x_{k}^{\prime 2}\right)
$$

where $x_{k}$ and $x_{k}^{\prime}$ are the position and angle of the bunch at the time when the kick is applied. The change of $\epsilon$ for a kick $\theta$ is then

$$
\triangle \epsilon=2 \pi \theta\left(\alpha x_{k}+\beta x_{k}^{\prime}\right)
$$

$x_{k}$ and $x_{k}^{\prime}$ can be obtained from the PUE information typically obtained from the previous turn. Using just the information at the PUE $\left(\begin{array}{c}x_{p} \\ x_{p}^{\prime}\end{array}\right)$, of which of course only $x_{p}$ can be measured, gives:

$$
\begin{gathered}
\left(\begin{array}{c}
x_{k} \\
x_{k}^{\prime}
\end{array}\right)= \\
\left(\begin{array}{cc}
\sqrt{\frac{\beta_{k}}{\beta_{p}}}\left(\cos (\varphi)+\alpha_{p} \sin (\varphi)\right) & \sqrt{\beta_{k} \beta_{p}} \sin (\varphi) \\
-\frac{1+\alpha_{k} \alpha_{p}}{\sqrt{\beta_{k} \beta_{p}}} \sin (\varphi)+\frac{\alpha_{p}-\alpha_{k}}{\sqrt{\beta_{k} \beta_{p}}} \cos (\varphi) & \sqrt{\frac{\beta_{p}}{\beta_{k}}}\left(\cos (\varphi)-\alpha_{k} \sin (\varphi)\right)
\end{array}\right)\left(\begin{array}{c}
x_{p} \\
x_{p}^{\prime}
\end{array}\right)
\end{gathered}
$$

Inserting into eq. 2.2 then gives:

$$
\triangle \epsilon=2 \pi \theta\left[\sqrt{\frac{\beta_{k}}{\beta_{p}}}\left(\alpha_{p} \cos (\varphi)+\sin (\varphi)\right) x_{p}+\sqrt{\beta_{k} \beta_{p}}(\cos (\varphi)) x_{p}^{\prime}\right]
$$


With $\left(\begin{array}{c}x_{p} \\ x_{p}^{\prime}\end{array}\right)$ expressed in terms of $\epsilon$ and phase $\psi$

$$
\begin{gathered}
x_{p}=\sqrt{\frac{\beta_{p} \varepsilon}{\pi}} \cos \psi \\
x_{p}^{\prime}=\sqrt{\frac{\epsilon}{\pi \beta_{p}}}\left(-\alpha_{p} \cos \psi-\sin \psi\right)
\end{gathered}
$$

the change of $\epsilon$ becomes:

$$
\Delta \epsilon=-2 \theta \sqrt{\pi \beta_{k} \epsilon}(\sin \varphi \cos \psi+\cos \varphi \sin \psi)=-2 \theta \sqrt{\pi \beta_{k} \epsilon} \sin (\varphi+\psi)
$$

If the kick in the strip line is made simply proportional to the reading of the PUE or $\theta=k x_{p}=k \sqrt{\frac{\beta_{p} \epsilon}{\pi}} \cos \psi$ the effective change of the emittance, obtained by averaging over the phase variable $\psi$, is

$$
\langle\triangle \epsilon\rangle=\frac{1}{2 \pi} \int_{0}^{2 \pi} \triangle \epsilon d \psi=-k \epsilon \sqrt{\beta_{k} \beta_{p}} \sin \varphi .
$$

$\varphi$ is the phase advance between the PUE location and the strip line location. If both are located at the same place $\varphi=2 \pi \nu$. Clearly maximum damping is then achieved for a fractional betatron tune of $n / 4$. In this case the damping time constant is $k \beta \epsilon$. If the PUE in the AGS is located downstream of the kicker (F20) in straight section G5 $\varphi=2 \pi \nu-\delta$, with $\delta \approx 60^{\circ}$. The optimal tune value is then 8.92 which is much closer to the standard operating point of the AGS at injection.

\section{Recursive Digital Filter}

A digital filter can be used to process the information from the PUE before it is used to control the size of the kick applied with the strip lines. Using digital pipeline delays the information of several subsequent turns can be included in the processing. Whereas a non-recursive filter only allows the inclusion of a limited number of turn information, a recursive filter by feeding back the output of the filter to the input automatically includes the information of a very large number of turns. A recursive part of the filter is therefore best suited for closed orbit subtraction where the average position of many turns needs to be determined.

In the following the response of a simple recursive filter is calculated. The output is added to the input after being delayed by one turn and multiplied by a constant $\kappa$ :

$$
\begin{gathered}
\left(\begin{array}{c}
x_{\text {out }} \\
x_{\text {out }}^{\prime}
\end{array}\right)=(1-\kappa)\left[\left(\begin{array}{c}
x_{p} \\
x_{p}^{\prime}
\end{array}\right)+\kappa \times\left(\begin{array}{c}
x_{p} \\
x_{p}^{\prime}
\end{array}\right)_{-1}+\kappa^{2} \times\left(\begin{array}{c}
x_{p} \\
x_{p}^{\prime}
\end{array}\right)_{-2}+\cdots\right] \\
=(1-\kappa)\left[\left(1+\kappa T^{-1}+\left(\kappa T^{-1}\right)^{2}+\cdots\right)\left(\begin{array}{c}
\tilde{x}_{p} \\
\widetilde{x}_{p}^{\prime}
\end{array}\right)\right]+\left(\begin{array}{c}
\bar{x}_{p} \\
\bar{x}_{p}^{\prime}
\end{array}\right) \\
=(1-\kappa)\left(1-\kappa T^{-1}\right)^{-1}\left(\begin{array}{c}
\tilde{x}_{p} \\
\widetilde{x}_{p}^{\prime}
\end{array}\right)+\left(\begin{array}{c}
\bar{x}_{p} \\
\bar{x}_{p}^{\prime}
\end{array}\right)
\end{gathered}
$$


where $T$ is the one-turn matrix at the location of the PUE:

$$
T=\left(\begin{array}{cc}
\cos (2 \pi \nu)+\alpha_{p} \sin (2 \pi \nu) & \beta_{p} \sin (2 \pi \nu) \\
-\frac{1+\alpha_{p}^{2}}{\beta_{p}} \sin (2 \pi \nu) & \cos (2 \pi \nu)-\alpha_{p} \sin (2 \pi \nu)
\end{array}\right)
$$

The factor $(1-\kappa)$ guarantees that the time independent part of $x_{\text {out }}$ is equal to the closed orbit $\bar{x}_{p}$. The response is then

$$
\begin{gathered}
\left(\begin{array}{c}
x_{\text {out }} \\
x_{\text {out }}^{\prime}
\end{array}\right)=\left(\begin{array}{c}
\bar{x}_{p} \\
\bar{x}_{p}^{\prime}
\end{array}\right)+\frac{(1-\kappa)}{1-2 \kappa \cos (2 \pi \nu)+\kappa^{2}} \times \\
\left(\begin{array}{cc}
1-\kappa\left(\cos (2 \pi \nu)+\alpha_{p} \sin (2 \pi \nu)\right) & -\kappa \beta_{p} \sin (2 \pi \nu) \\
\kappa \frac{1+\alpha_{p}^{2}}{\beta_{p}} \sin (2 \pi \nu) & 1-\kappa\left(\cos (2 \pi \nu)-\alpha_{p} \sin (2 \pi \nu)\right)
\end{array}\right)\left(\begin{array}{l}
\tilde{x}_{p} \\
\widetilde{x}_{p}^{\prime}
\end{array}\right) .
\end{gathered}
$$

For $\kappa=0$ the filter has no effect. For $\kappa \rightarrow 1, x_{\text {out }}$ becomes equal to the closed orbit, averaging over an infinite number of turns. For intermediate values of $\kappa$ the determination of the closed orbit is obtained from a limited number of turns. In fact, by adjusting $\kappa$ the response time of the damper to changes in the closed orbit can be adjusted. For example, injection error damping benefits from a fast response time whereas damping of transverse instabilities benefits from a much slower response time which then allows for a tune value much closer to an integer, as is outline later. A implementation for a simple adjustment of $\kappa$ is described later. Also, the filter determines the closed orbit for each bunch separately due to the pipe lined structure of the delay.

To obtain a value for the strip line kick that does not depend on the closed orbit the output of the recursive filter has to be subtracted from the direct PUE signal:

$$
\begin{gathered}
\theta=k\left(x_{p}-x_{\text {out }}\right) \\
=k\left(\widetilde{x}_{p}-\frac{(1-\kappa)}{1-2 \kappa \cos (2 \pi \nu)+\kappa^{2}}\left(\left(1-\kappa\left(\cos (2 \pi \nu)+\alpha_{p} \sin (2 \pi \nu)\right)\right) \widetilde{x}_{p}-\kappa \beta_{p} \sin (2 \pi \nu) \widetilde{x}_{p}^{\prime}\right)\right) \\
=k \sqrt{\frac{\beta_{p} \epsilon}{\pi}}\left(\cos \psi-\frac{(1-\kappa)}{1-2 \kappa \cos (2 \pi \nu)+\kappa^{2}}(\cos \psi+\kappa(\sin (2 \pi \nu) \sin \psi-\cos (2 \pi \nu) \cos \psi))\right)
\end{gathered}
$$

Inserting into Eq. 2.6 and averaging over the phase $\psi$ then gives:

$$
\begin{gathered}
\langle\triangle \epsilon\rangle= \\
-k \epsilon \sqrt{\beta_{k} \beta_{p}}\left(\sin \varphi-\frac{(1-\kappa)}{1-2 \kappa \cos (2 \pi \nu)+\kappa^{2}}(\sin \varphi(1-\kappa \cos (2 \pi \nu))+\cos \varphi \kappa \sin (2 \pi \nu))\right)
\end{gathered}
$$

Fig. 1 shows the block diagram for this filter algorithm. Fig.2 shows the same filter where the multiplications have been implemented with simple $n$-bit shift registers and an additional adder[?]. $\kappa$ is related to $n$ by the formula

$$
\kappa=\frac{2^{n}-1}{2^{n}}
$$


Not all values for $\kappa$ can be realized with this implementation. But the value of $\kappa$ is not very critical as it only determines the integration range for the closed orbit subtraction. Fig. 3 and 4 show the responses of the filter as a function of tune for $\varphi=2 \pi \nu-\delta$, with $\delta \approx 60^{\circ}$ and $\varphi=2 \pi \nu$, respectively, for $n=1,2,3,4,5$ or $\kappa=\frac{1}{2}, \frac{3}{4}, \frac{7}{8}, \frac{15}{16}, \frac{31}{32}$.

\section{Notch Filter with Recursive Path}

By adding an additional one-turn delay in the recursive path of the filter, the filter effectively becomes a notch filter for $\kappa=0$ but changes only little for large values of $\kappa$. However, the filter can be implemented with much fewer components as will be shown later. The block diagram is shown in Fig. 5 and the implementation is shown in Fig. 6. The result of the upper recursive path in Fig. 5 is

$$
\begin{gathered}
\left(\begin{array}{c}
x_{\text {out }} \\
x_{\text {out }}^{\prime}
\end{array}\right)=\left(\begin{array}{c}
\bar{x}_{p} \\
\bar{x}_{p}^{\prime}
\end{array}\right)+(1-\kappa)\left(1-\kappa T^{-1}\right)^{-1} T^{-1}\left(\begin{array}{c}
\widetilde{x}_{p} \\
\widetilde{x}_{p}^{\prime}
\end{array}\right) \\
=\left(\begin{array}{c}
\bar{x}_{p} \\
\bar{x}_{p}^{\prime}
\end{array}\right)+\frac{(1-\kappa)}{1-2 \kappa \cos (2 \pi \nu)+\kappa^{2}} \times \\
\left(\begin{array}{cc}
\left(\cos (2 \pi \nu)-\alpha_{p} \sin (2 \pi \nu)\right)-\kappa & -\beta_{p} \sin (2 \pi \nu) \\
\frac{1+\alpha_{p}^{2}}{\beta_{p}} \sin (2 \pi \nu) & \left(\cos (2 \pi \nu)+\alpha_{p} \sin (2 \pi \nu)\right)-\kappa
\end{array}\right)\left(\begin{array}{c}
\widetilde{x}_{p} \\
\widetilde{x}_{p}^{\prime}
\end{array}\right) .
\end{gathered}
$$

Subtracting $x_{\text {out }}$ from the reading of the PUE again allows to perform the closed orbit subtraction:

$$
\begin{gathered}
\theta=k\left(x_{p}-x_{\text {out }}\right) \\
=k\left(\tilde{x}_{p}-\frac{(1-\kappa)}{1-2 \kappa \cos (2 \pi \nu)+\kappa^{2}}\left(\left(\left(\cos (2 \pi \nu)-\alpha_{p} \sin (2 \pi \nu)\right)-\kappa\right) \tilde{x}_{p}-\beta_{p} \sin (2 \pi \nu) \widetilde{x}_{p}^{\prime}\right)\right) \\
=k \sqrt{\frac{\beta_{p} \epsilon}{\pi}}\left(\cos \psi-\frac{(1-\kappa)}{1-2 \kappa \cos (2 \pi \nu)+\kappa^{2}}(\sin (2 \pi \nu) \sin \psi+\cos (2 \pi \nu) \cos \psi-\kappa \cos \psi)\right)
\end{gathered}
$$

Inserting into Eq. 4.1 and averaging over the phase $\psi$ then gives:

$$
\begin{gathered}
\langle\triangle \epsilon\rangle= \\
-k \epsilon \sqrt{\beta_{k} \beta_{p}}\left(\sin \varphi-\frac{(1-\kappa)}{1-2 \kappa \cos (2 \pi \nu)+\kappa^{2}}(\sin \varphi(\cos (2 \pi \nu)-\kappa)-\cos \varphi \sin (2 \pi \nu))\right)
\end{gathered}
$$

Fig. 7 and 8 show the responses of this filter as a function of tune for $\varphi=2 \pi \nu-\delta$, with $\delta \approx 60^{\circ}$ and $\varphi=2 \pi \nu$, respectively, for $n=0,1,2,3,4$, or $\kappa=0, \frac{1}{2}, \frac{3}{4}, \frac{7}{8}, \frac{15}{16}$.

\section{Conclusion}

The digital notch filter with a recursive path for closed orbit subtraction offers an efficient and flexible way to obtain fast damping at a high tune value close to an integer value and still have reliable subtraction of the closed orbit information. As Fig. 8 shows full damping efficiency can be achieved at betatron tune of 8.9 with $n=4$. 


\section{Figure Captions}

Fig. 1 Block diagram of the digital filter with recursive path for closed orbit subtraction.

Fig. 2 Block diagram of filter from Fig. 1 implemented with n-bit shift register.

Fig. 3 Response function for digital filter from Fig. 1 for $n=1,2,3,4,5$ and $\varphi=2 \pi \nu$.

Fig. 4 Response function for digital filter from Fig. 1 for $n=1,2,3,4,5$ and $\varphi=$ $2 \pi \nu-\delta$, with $\delta \approx 60^{\circ}$.

Fig. 5 Block diagram of digital notch filter with recursive path for closed orbit subtraction.

Fig. 6 Block diagram of filter from Fig. 5 implemented with n-bit shift register.

Fig. 7 Response function for digital notch filter from Fig. 5 for $n=0,1,2,3,4$ and $\varphi=2 \pi \nu$.

Fig. 8 Response function for digital notch filter from Fig. 5 for $n=0,1,2,3,4$ and $\varphi=2 \pi \nu$, with $\delta \approx 60^{\circ}$.

\section{References}

[1] Transverse Damper Algorithms, T. Roser, AGS/AD/Tech Note No. 377 (1993)

[2] Damping of Phase Errors at Injection in the LHC, D. Boussard and E. Onillon, Proc. of the 1993 Particle Accelerator Conference, p.2379, Washington, DC. 1993 


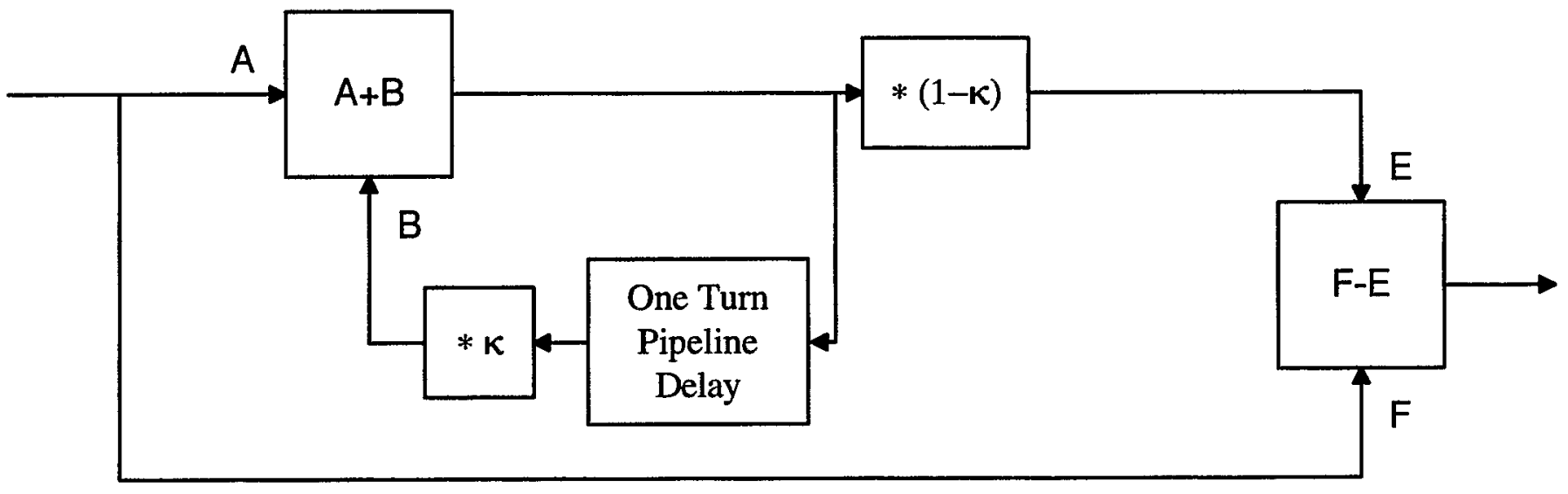

Fig. 1. Block diagram of digital filter with recursive path for closed orbit subtraction.

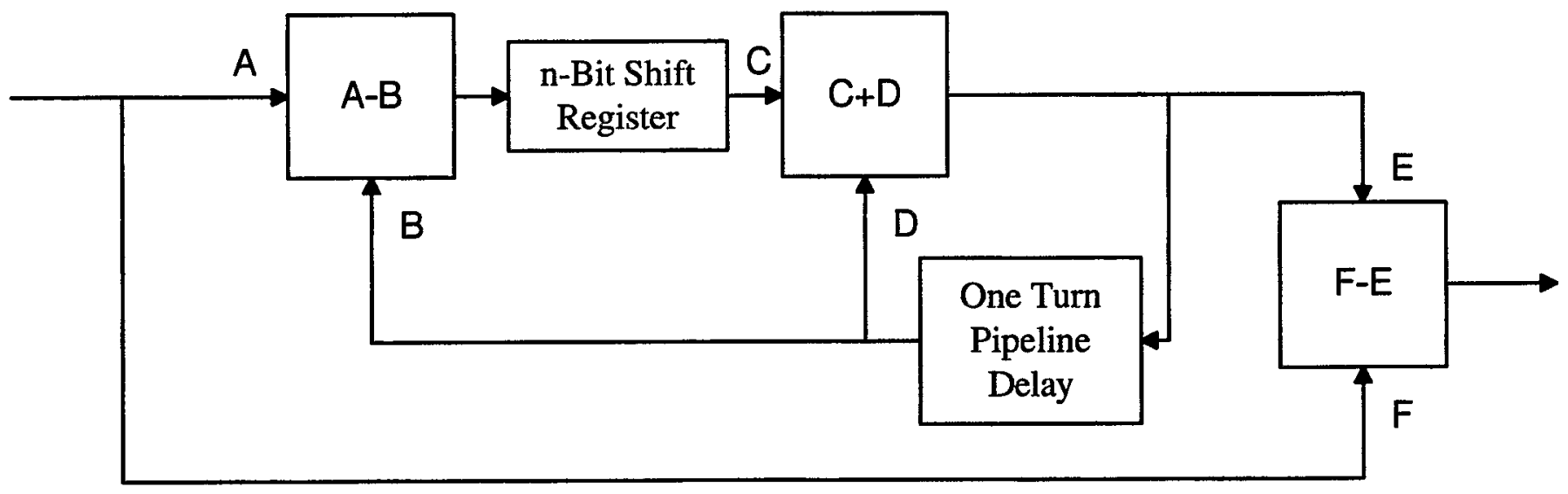

Fig. 2. Block diagram of filter from Fig. 1 implemented with n-bit shift registers. 


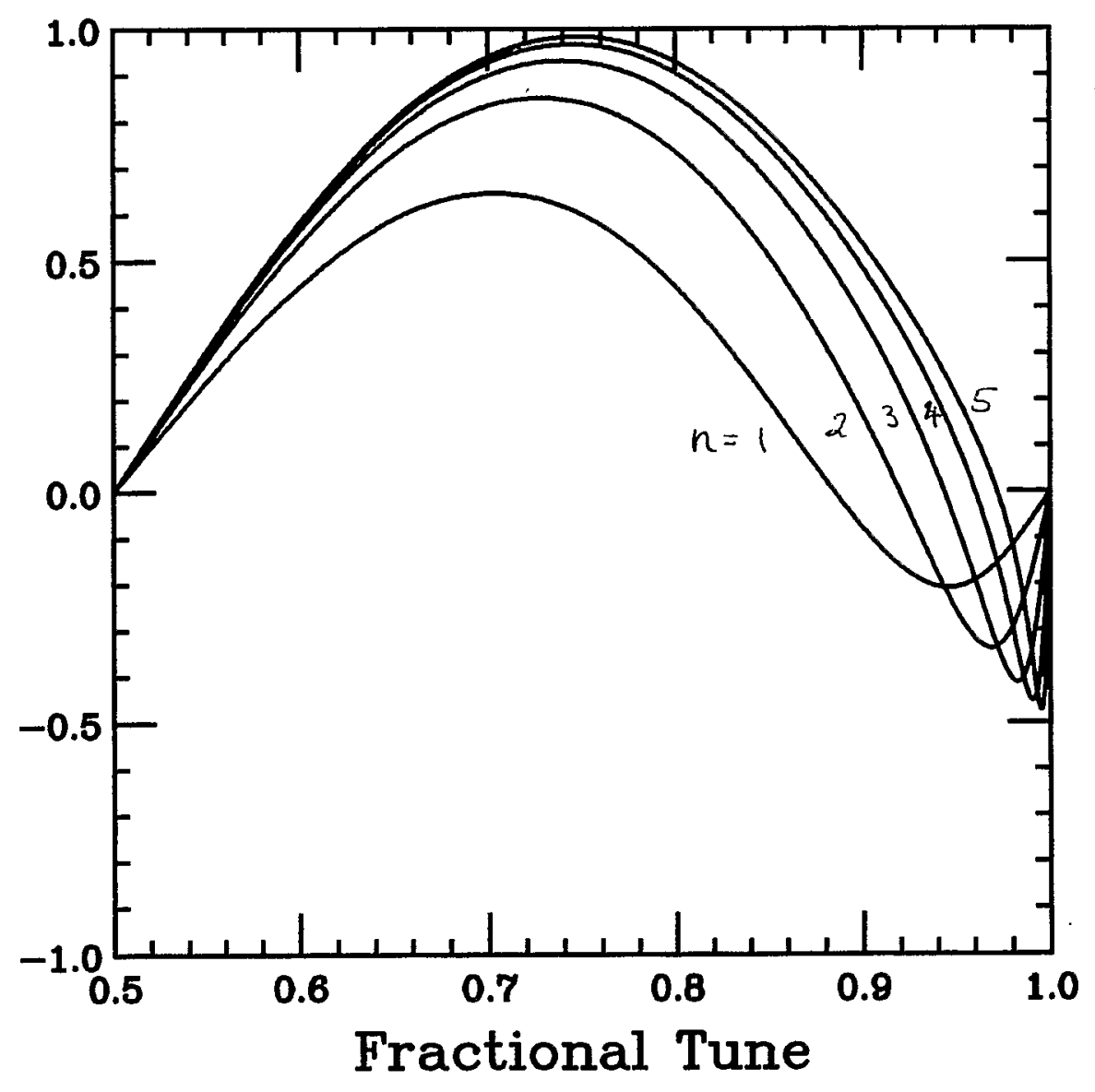

Fig. 3 


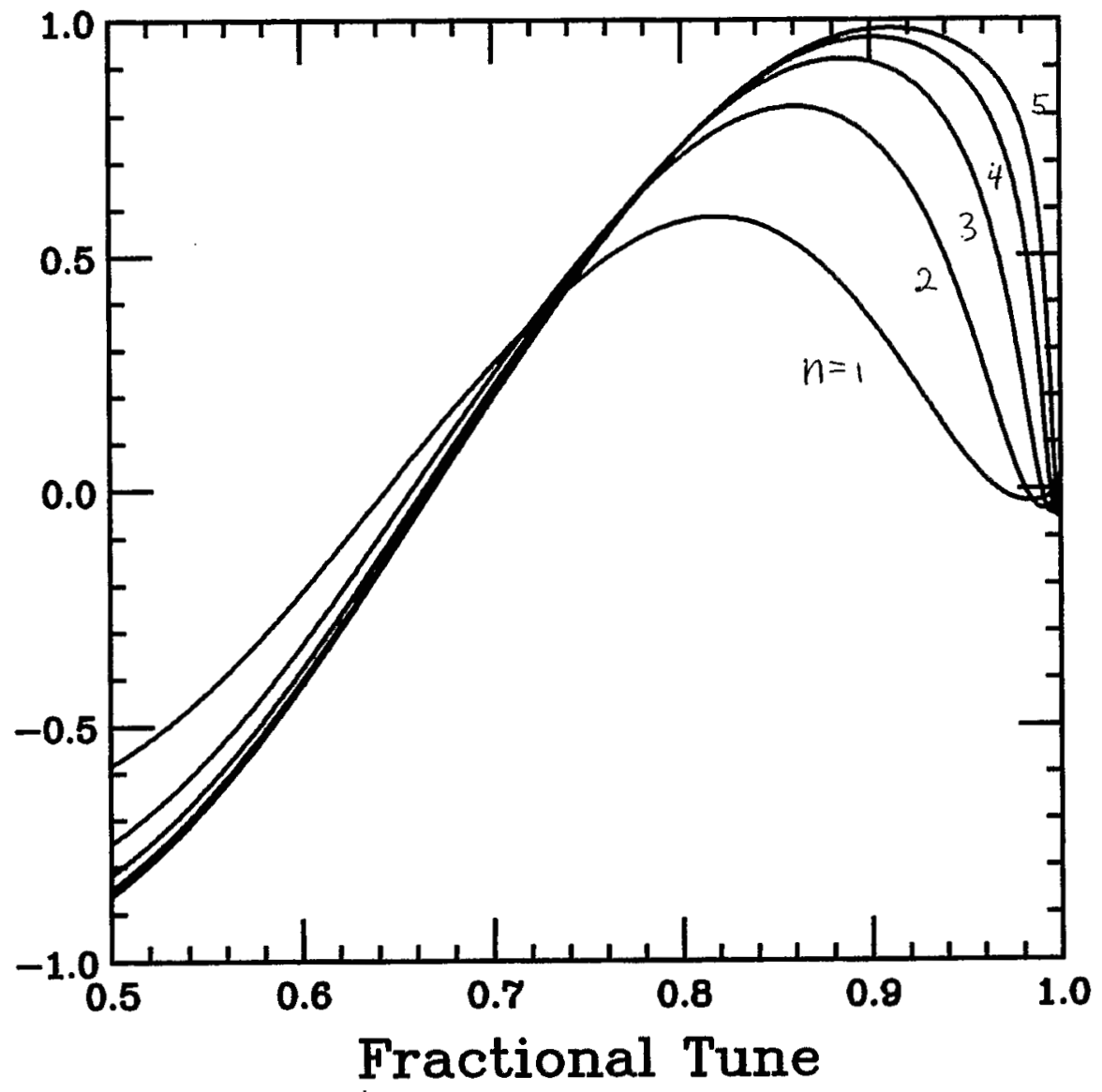

Fig. 4 


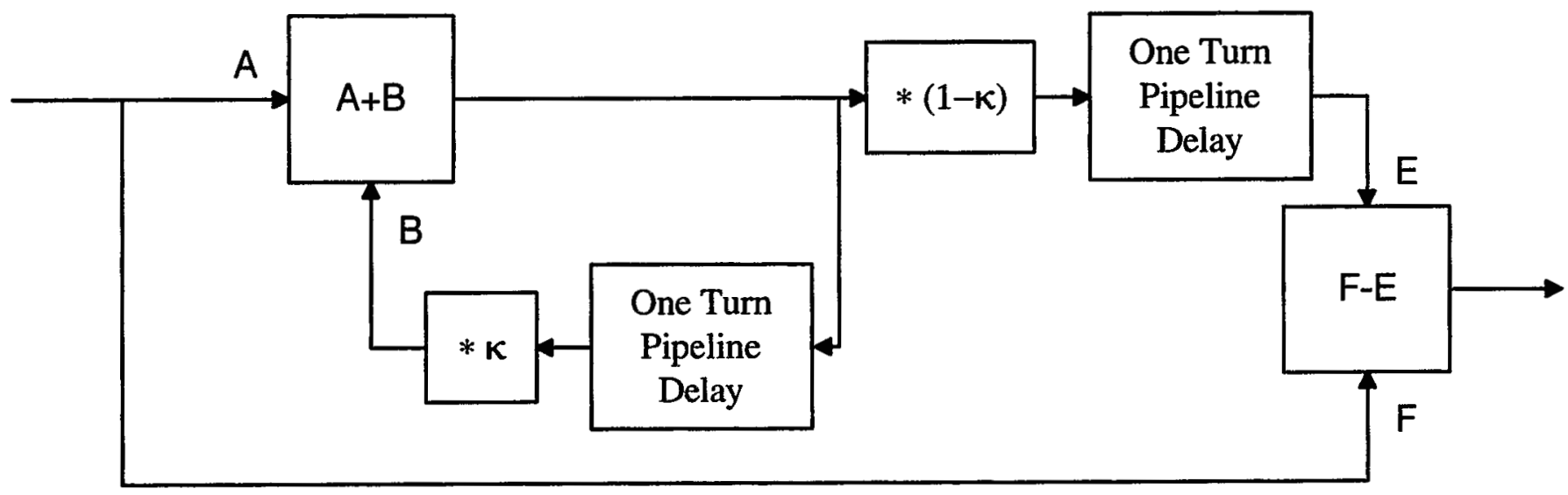

Fig. 5. Block diagram of digital notch filter with recursive path for closed orbit subtraction.

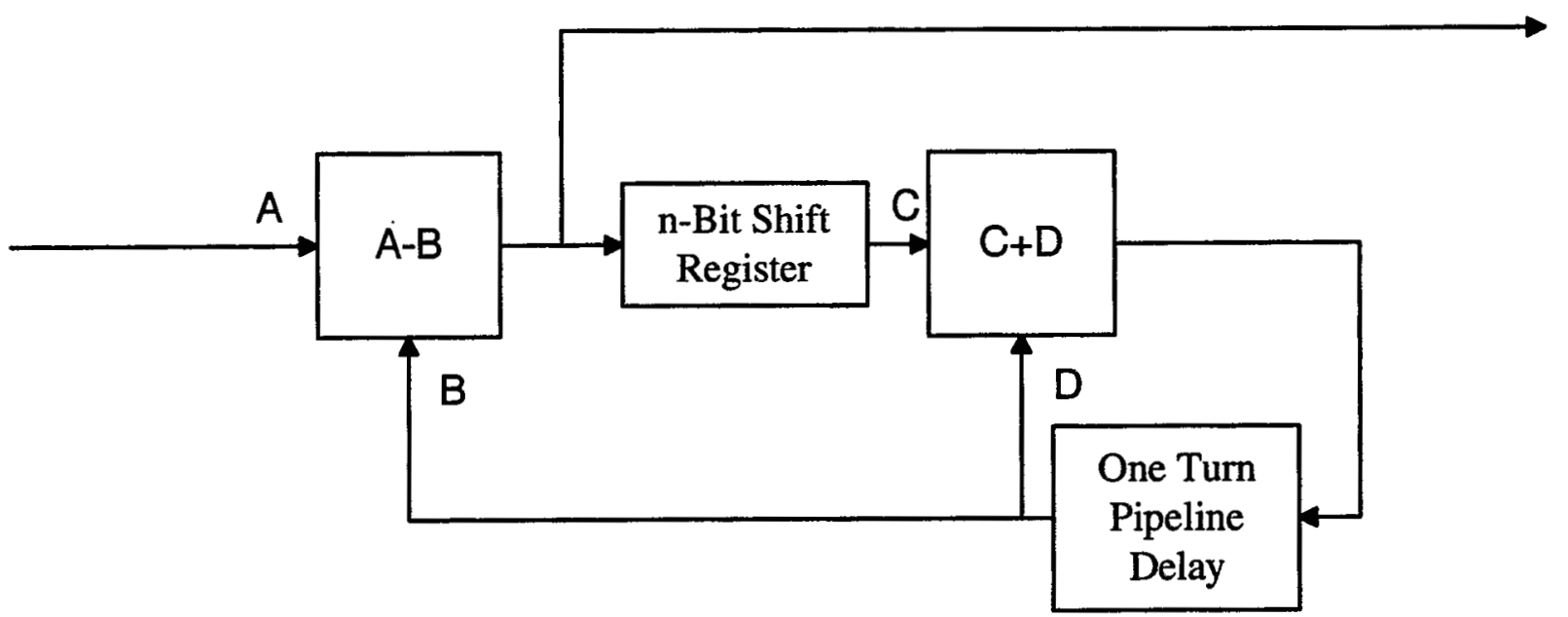

Fig. 6. Block diagram of filter of Fig. 5 implemented with n-bit shift register. 


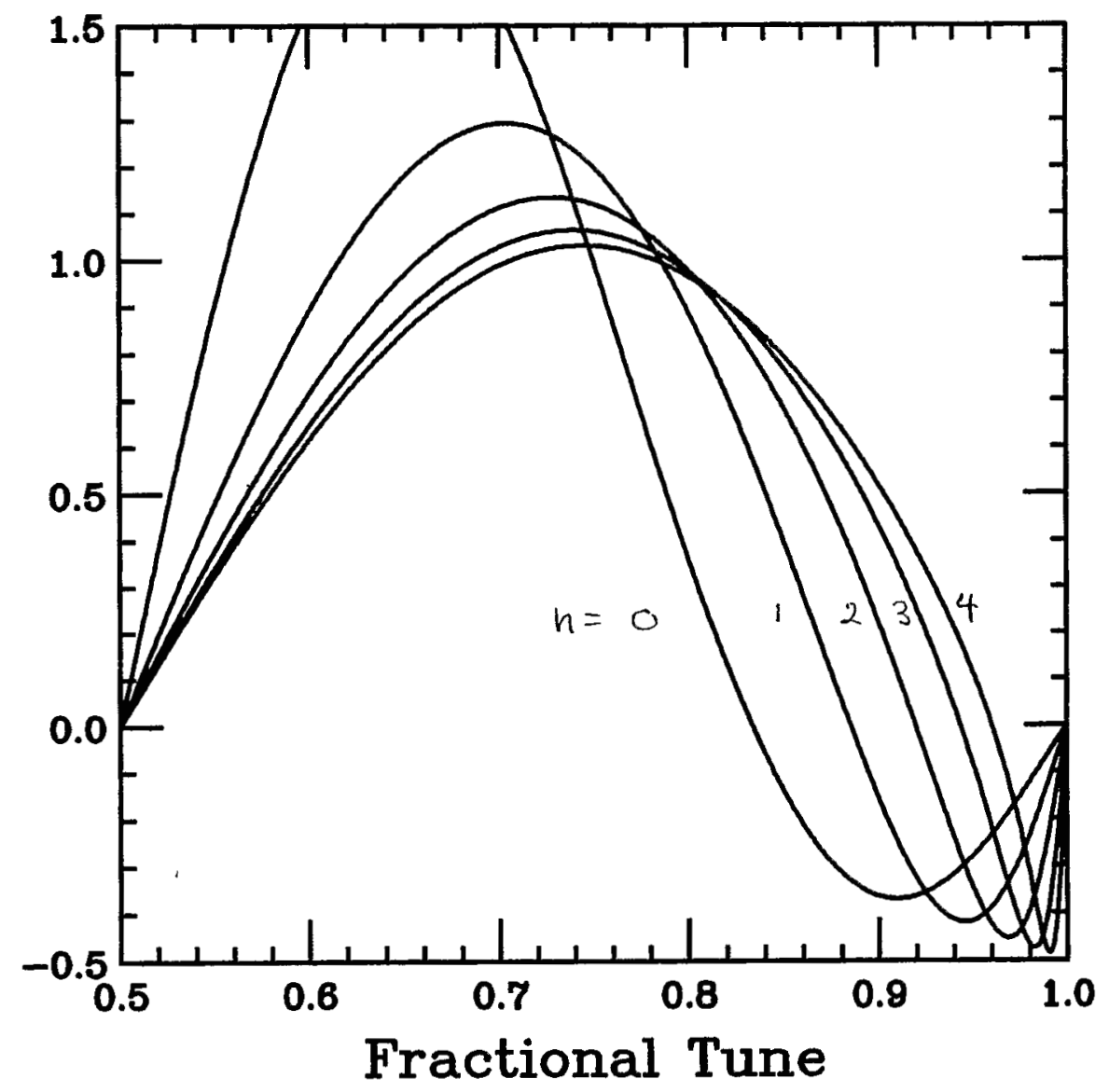

Fig 7 


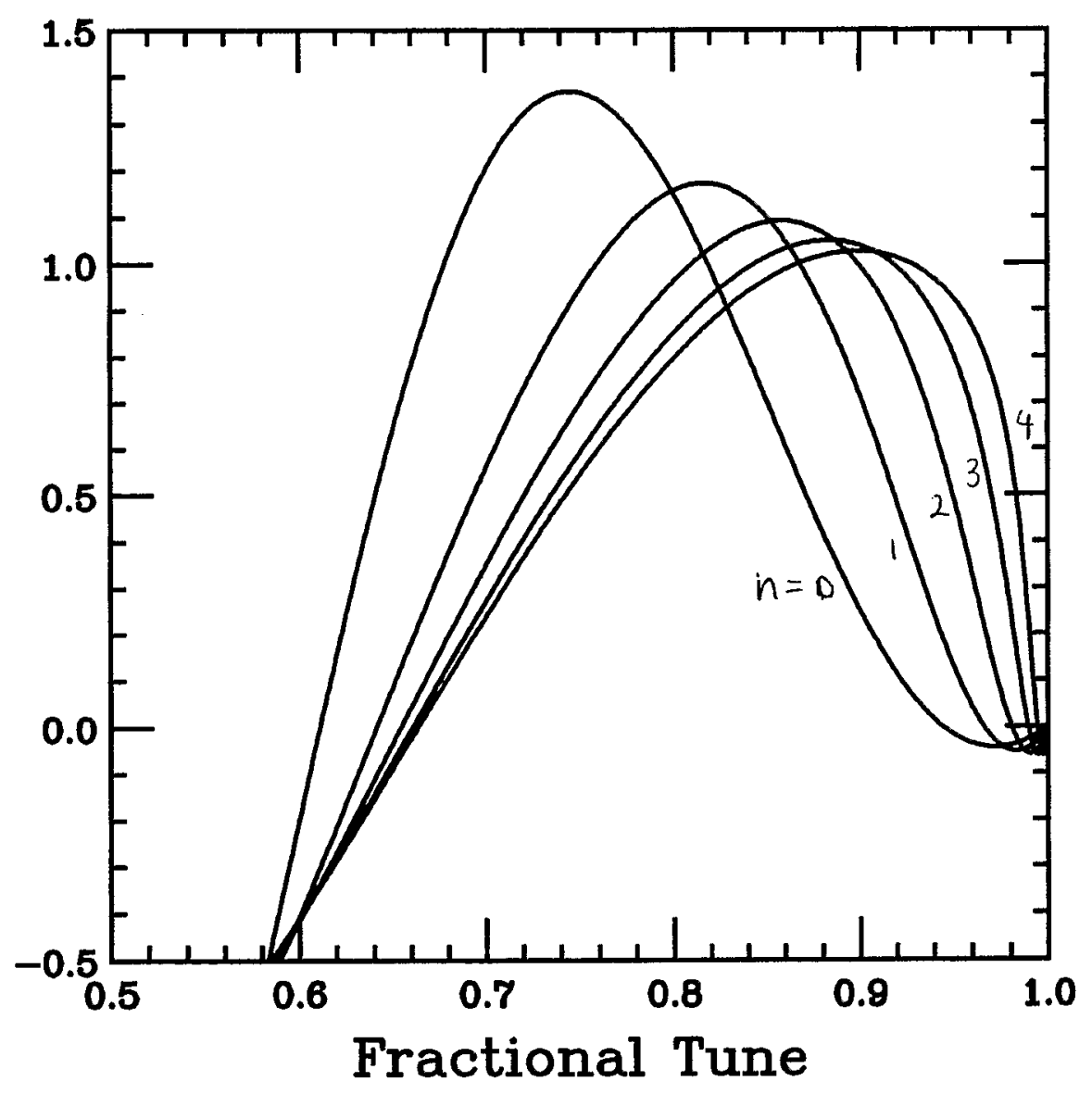

Fig. 8 